\title{
Phytotherapeutikum senkt die Rezidivrate bei Harnwegsinfekten
}

_ Unkomplizierte Harnwegsinfektionen sind nach Atemwegsinfekten der häufigste Grund für Antibiotikaverschreibungen und gehören zu den am meisten diagnostizierten Infektionen im ambulanten Bereich. Die aktuelle S-3-Leitlinie merkt an, dass eine Behandlung mit Antibiotika zur schnelleren Beschwerdelinderung bei einer akuten unkomplizierten Zystitis führt [S-3-Leitlinie Harnwegsinfektionen. 2010 AWMF-RegisterNr. 043/044]. Die zunehmende Resistenzentwicklung macht jedoch einen überlegten Einsatz der Medikamente notwendig. Die Leitlinie legt nahe, dass eine symptomatische Therapie bei unkomplizierten Harnwegsinfektionen eine vertretbare Alternative zur Antibiose sein kann, ohne dass eine erhöhte Gefahr von Komplikationen zu befürchten ist. Hier kann das Phytotherapeutikum Canephron ${ }^{\circledR} \mathrm{N}$ hilfreich sein. Es hat sich bei Heranwachsenden ab zwölf Jahren und Erwachsenen zur Behandlung von akuten und rezidiverenden Infektionen der unteren Harnwege sowie von Symptomen der Reizblase bewährt. Die pflanzliche Dreierkombination aus Rosmarinblättern, Tausendgüldenkraut und Liebstöckelwurzel besitzt ein breites pharmakologisches Wirkprofil und wirkt antibakteriell, antiphlogistisch, spasmolytisch und di- uretisch. Dadurch werden die unangenehmen Beschwerden wie Krämpfe und Brennen beim Wasserlassen gelindert und die Keime werden wirksam bekämpft und ausgespült.

Canephron ${ }^{\circledast} \mathrm{N}$ kann auch in Kombination mit einer antibiotischen Therapie eingesetzt werden, wie eine Studie an Probanden mit Harnwegsinfektionen und begleitendem Diabetes mellitus/Metabolischem Syndrom zeigte [Ivanov et al. 2004]. Patienten mit dieser Grunderkrankung leiden circa 20-25 mal häufiger an einem Harnwegsinfekt als gesunde Patienten und sind daher besonders auf eine Rezidivprophylaxe angewiesen. Das Phytotherapeutikum wurde in der Verumgruppe akut zum Antibiotikum und anschließend drei Monate zur Rezidivprophylaxe gegeben. Die Kontrollgruppe wurde nur akut mit einem Antibiotikum behandelt.

Canephron ${ }^{\circledast} \mathrm{N}$ als add-on führte während der Akuttherapie zu einer schnelleren Symptomlinderung als das Antibiotikum allein. Die dreimonatige prophylaktische Einnahme von Canephron ${ }^{\circledR} \mathrm{N}$ senkte die Rezidivrate um $73 \%$ (von 21,7\% auf 5,9\%). Aufgrund seiner guten Verträglichkeit zeigte es sich auch für die längerfristige Anwendung geeignet.

Nach Informationen von Bionorica, Neumarkt

\section{Axitinib erhält EU-Zulassung zur Zweitlinientherapie des Nierenzellkarzinoms}

— Die Europäische Arzneimittelagentur EMA hat Axitinib (Inlyta ${ }^{\oplus}$ ) die Zulassung zur Therapie von erwachsenen Patienten mit fortgeschrittenem Nierenzellkarzinom (mRCC) nach Versagen einer vorangegangenen Therapie mit Sutent ${ }^{\circledR}$ (Sunitinib) oder einem Zytokin erteilt. Der Kinase-Inhibitor Axitinib ist eine orale Therapie, die auf die selektive Hemmung der VEGF-Rezeptoren (Vascular Endothelial Growth Factor Receptors) 1, 2 und 3 ausgerichtet ist. Die Zulassung der Europäischen Kommission basiert auf den Daten der Phase-III-Studie AXIS, in der Axitinib das progressionsfreie Überleben (PFS) mit einem medianen PFS von 6,8 Monaten im Vergleich zu 4,7 Monaten unter Sorafenib signifikant verlängerte (Hazard Ratio $=0,67$, $p<0,0001$ ) [Summary of Product Characteristics for INLYTA. Sandwich, Kent: UK; 2012]. „Wir freuen uns über die Entscheidung der Europäischen Kommission, Inlyta ${ }^{\circledR}$ für erwachsene Patienten mit mRCC zuzulassen, deren Erkrankung nach Behandlung mit Sutent ${ }^{\oplus}$ oder einem Zytokin fortschreitet. mRCC ist eine komplexe Erkrankung und Pfizer Oncology setzt sich dafür ein, Ärzten und Patienten neue zielgerichtete Medikamente zur Verfügung zu stellen", sagt Dr. Andreas Penk, Vorsitzender der Geschäftsführung von Pfizer Deutschland. Inlyta sei eine wichtige Ergänzung des mRCC-Medikamenten-Portfolios von Pfizer Oncology.

Nach Informationen von Pfizer, Berlin
Optimierter Einmal-Katheter

Wer selbst katherterisieren muss, möchte dies diskret, unkompliziert und sicher erledigen. Der Simplicath ${ }^{\circledR}$-Einmal-Katheter wurde daher im intensiven Austausch mit Betroffenen optimiert. Die neue Verpackung sorgt dafür, dass der Katheter ungeknickt bleibt und deshalb auch für Männer leichter einzuführen ist. Dank neuer Flaschenform des Systems mit Beutel stört kein unnötiges Material im oberen Bereich. Eine gleichmäßige Gelbeschichtung sorgt für hohe Gleitfähigkeit. Griffmulde und Halteösen erleichtern die Handhabung von Katheter und Urinbeutel. Wichtigster Vorteil ist der sichere Schutz vor Harnwegsinfektionen: Die Vorlaufspitze verhindert, dass die Katheterspitze mit dem keimbelasteten Harnröhreneingang in Kontakt kommt. Die Möglichkeit zur Anforderung eines Simplicath ${ }^{\circledR}$-Musters oder der neuen Produktbroschüre für Ärzte und Pflegekräfte bzw. der Patientenbroschüre besteht unter www.simplycath.de. oder per E-Mail unter simplicath@uromed.de.

Nach Informationen von

UROMED, Oststeinbek

Schonende Lasertherapie bei Hämorrhoiden

Am Anfang eines Hämorrhoidalleidens haben die Betroffenen kaum Beschwerden. Deshalb ist es wichtig, bereits die ersten Symptome zu erkennen um eine beschwerliche Operation zu vermeiden. Typische Symptome am Anfang sind Juckreiz und Brennen im Afterbereich, Schmerzen, Blut im Stuhl und Nässen. Nun sollte gehandelt werden. Die innovative minimalinvasive LHP $^{\text {TM }}$ Lasertherapie der biolitec ${ }^{\oplus}$ ermöglicht die ambulante und schmerzarme Behandlung von Hämorrhoiden mittels Laserverödung. Die Hämorrhoiden werden unter geeigneter Anästhesie über einen kleinen Einstich mit einer dünnen Lasersonde von innen heraus geschrumpft ohne das Anoderm zu verletzen. Es sind keine Schnitte und Nähte notwendig und es entstehen keine offenen Wunden. Die Gefahr einer Infektion ist dadurch vermindert. Durch die gezielte Tiefenwirkung des Lasers werden die vergrößerten Hämorrhoiden zusätzlich von ihrer Blutversorgung abgeschnitten. Nach der Laserbehandlung beginnen die Hämorrhoiden nach kurzer Zeit zu schrumpfen.

Nach Informationen von

biolitec, Jena 